Demografischer Wandel in der Wirtschaft

\section{Warum Unternehmen ältere Arbeitnehmer brauchen}

\author{
Die Anforderungen an Arbeitnehmer verändern sich immer \\ schneller. Bisher wurden die passenden Menschen ausgewählt, \\ weil das Angebot groß genug war. Aber diese Strategie geht kaum \\ noch auf. Deshalb beginnt ein Umdenken, hin zu einer gezielten \\ Integrationsleistung in der Personalentwicklung. Von Siegmar \\ Otto und Sven Voelpel
}

$\mathbf{N}$ ach meiner vorzeitigen Pensionierung mit 61 Jahren habe ich nach einer Interimsposition als Projektleiter gesucht. Es war für mich eine große Überraschung, dass mich Lonza - Weltmarktführer in der Produktion und Prozessbegleitung von pharmazeutischen Wirkstoffen im chemischen und biotechnologischen Bereich - auch in diesem Alter ohne Wenn und Aber in der Konzernfunktion Global Human Resources fest angestellt hat. Dies mit dem Hinweis, dass für einen Job bei Lonza nur die Qualifikation ausschlaggebend ist und andere Faktoren wie Alter, Nationalität und Geschlecht irrelevant sind. Weiter ist sich Lonza bewusst, dass der demografische Wandel in den kommenden Jahren weitreichende Herausforderungen an die Firma stellen wird und deshalb neue Denk- und Sichtweisen erfordert.

Alfred Zimmerli, 62, Human Resources, Lonza

Das Beispiel des Unternehmens Lonza ist immer noch als Ausnahme zu bewerten. Nur wenigen gelingt die koordinierte, zufriedenstellende und damit meistens gewinnbringende Integration älterer Arbeitnehmer. Daneben gibt es eine ganze Reihe von Projekten, die sich an ähnlichen Best-Practice-Beispielen orientieren, aber aufgrund mangelhafter Rahmenbedingungen scheitern oder im Sande verlaufen. Es wird oft nicht auf die spezifischen Begleitumstände eingegangen, sodass unangepasste, zu allgemeine Instrumente nicht zum erwünschten Erfolg führen. Entsprechend gestaltet sich der Forschungsstand zum Themenbe- reich. In den akademischen, politischen und wirtschaftlichen Bereichen wird an einer beachtlichen Reihe von Projekten gearbeitet, um die Auswirkungen der alternden Belegschaft zu untersuchen, um Vorschläge für verschiedene Interventionsmaßnahmen zu entwickeln und diese umzusetzen. Es ist dies ein Feld, in dem der Leidensdruck der Wirtschaft im Vergleich zur praktisch anwendbaren und wissenschaftlich abgesicherten Forschung relativ hoch ist. Dies führt zu vielen, von der Wirtschaft geförderten Projekten. Aber wegen des fehlenden fundierten theoretischen Rahmens bleibt es oft bei einzelnen Anstrengungen ohne den wünschenswerten intensiven Austausch (Kistler 2007).

\section{Erfolgreiche Integration verlangt nach spezifischen Instrumenten}

Voranschreiten hieße in diesem Fall, auf die vorhandenen Kooperationen zwischen Unternehmen und der Wissenschaft aufzubauen und auch einen wirklichen Austausch stattfinden zu lassen. Dies bedarf einer Übersetzungsleistung zwischen den beiden Systemen, wie sie im Fall von Corporate Social Responsibility explizit mit Change agents propagiert wird (Cramer 2006; Dover 2003). Aus erkenntnistheoretischer Perspektive müsste der Untersuchungsbereich ausgedehnt werden. Es sind wesentlich tiefgründigere Analysen der Rahmenbedingungen notwendig und auch die Eva- luation von Projekten sollte idealerweise formativ sein und einen ausreichenden Zeitraum abdecken (Bamberger 2006).

Weiterhin hat der globale Kontext einen erheblichen Einfluss auf die hier besprochene Forschung. Wie entwickeln sich zum Beispiel die Bevölkerungsströme? In welche Richtung wandern Fachkräfte? Steigen Konsum und Absatz wie im bisher gekannten Maßstab? Brauchen wir in Zukunft tatsächlich so viele Arbeitskräfte? Was ist mit dem technologischen Fortschritt und den damit verbundenen Risiken und Vorteilen? Wird sich der Konsum dematerialisieren, wie es für eine nachhaltige Entwicklung sehr förderlich sein könnte (Linz 2006)? Dies sind nur einige Fragen bezüglich des Konzeptes nachhaltiger Entwicklung mit Auswirkungen auf den Arbeitsmarkt und der damit verknüpften Strategien wie intra- und intergenerationeller Gerechtigkeit, Effizienz, Suffizienz oder Konsistenz. Dabei ist zu beachten, dass nachhaltige Entwicklung für verschiedene Akteure unterschiedliche Bedeutungen mit sich führt (Otto 2007).

Welche konkreten inhaltlichen Fragestellungen sind es jedoch, die in diesem Bereich von den Forschern bearbeitet werden sollen? Dazu einige praxisrelevante Kernfragen:

- Wie groß und wie ernst sind die Herausforderungen der demografischen Entwicklung?

- Warum sind die Herausforderungen gerade heute kritisch?

- Welche Unternehmen reagieren bereits?

【 Können die Herausforderungen bewältigt werden?

- Was sind die Lösungen für ein Unternehmen?

\section{Der Verlust kritischen Wissens}

Die Herausforderungen des demografischen Wandels für Unternehmen sind umfassend und sehr ernst. Der Verlust kritischen Wissens und eine zunehmende Knappheit gut ausgebildeter Arbeitnehmer sind dabei die Hauptprobleme. Diese Herausforderungen sind jetzt, sind 
heute kritisch, wenn man sich die unumstößlichen Trends vor Augen führt: alternde Bevölkerungen, Erwerbsbevölkerungen und Belegschaften, Vorherrschaft traditioneller Verrentungspraktiken, steigender Innovationsdruck und neue Denkweisen bezüglich Arbeit und Sinn des Lebens innerhalb der Gesellschaft. Die Herausforderungen können gemeistert werden, allerdings setzt dies drei wichtige Dinge voraus: ein Verständnis der Wechselwirkungen zwischen den einzelnen Herausforderungen, ein Verständnis für die Auswirkungen des demografischen Wandels auf das Geschäftsmodell von Unternehmen sowie strategisch-operationale Fähigkeiten bezüglich der Handlungsfelder, mit denen man der skizzierten Entwicklung entgegentreten kann (Voelpel 2007).

In ihrem Buch „Herausforderung 50 plus" liefern die Autoren mit den fünf Handlungsfeldern - neue Denkweisen im Management, Unterstützung neuer Prozesse des Wissensmanagements, Gestaltung einer angemessenen Arbeitsumgebung, Einführung neuer Prozesse im Gesundheitsmanagement sowie neue Praktiken und Werkzeuge im Human Resources Management - einen entsprechenden wissenschaftlichen Rahmen zur Bearbeitung der aufgezeigten Fragestellungen.

Kreativität und Innovationsfähigkeit werden gesteigert, wenn die Denkweisen der Verantwortlichen sich ändern und dadurch die Bereitschaft gestärkt wird, Dinge anders oder besser zu machen oder sogar ganz neue Wege zu gehen. Neue Denkweisen sind eine wichtige Voraussetzung, um die richtigen Wissensmanagementprozesse, Arbeitsumgebungen sowie ergonomischen Werkzeuge und Technologien zu entwickeln und umzusetzen.

Wissensmanagementprozesse, die Bewahrung, Vergrößerung, Verjüngung, Wiedergewinnung und die Weitergabe von Wissen, sind auf entsprechende $\mathrm{Hu}$ man-Resources-Management-Methoden angewiesen. Diese Prozesse bedürfen einer Unterstützung durch die richtige Arbeitseinstellung und physische Leistung älterer Mitarbeiter, einer förderlichen Arbeitsumgebung und Unternehmenskul- tur, des richtigen Führungsstils und gezielt eingesetzter physisch unterstützender Einrichtungen.

Das betriebliche Gesundheitsmanagement ist ein weiterer Schlüssel zum erfolgreichen Management alternder Belegschaften. Es ist eng verzahnt mit den notwendigen Bewusstseinsveränderungen und neuen Denkweisen, der physischen Leistungsfähigkeit der Mitarbeiter, der Arbeitssicherheit und Arbeitsgestaltung, Wissensmanagementprozessen und Human-Resources-Praktiken. Gesundheit sollte zudem nicht nur als rein physische Angelegenheit betrachtet werden, sondern als ganzheitliches Konzept auch mentale beziehungsweise psychische Aspekte einbeziehen. Dies gilt vor allem im Hinblick auf die Zunahme stressbedingter, psychosomatischer Erkrankungen.

\section{Neuausrichtung der Personalentwicklung}

Human-Resources-Management-Prozesse und -Praktiken sind reif für eine grundlegende Neuausrichtung, wenn sie sich als nützliche Tools zur Bewältigung der demografischen Herausforderungen bewähren sollen. In einer wissensvernetzten Innovationsökonomie, in der westliche Konzerne mehr und mehr mit alternden Belegschaften zurechtkommen müssen, ist das Human Resources Management gezwungen, sich den anderen vier Handlungsfeldern zu öffnen und sie mit sich und untereinander zu vernetzen.

Die bisher beschriebenen Handlungsfelder sollten in ihrer Summe erheblich positive Auswirkungen auf die Arbeitsumgebung haben. Dabei ist eine zusätzliche bewusste Arbeitsplatzgestaltung im Rahmen der angeführten Verbesserungen ebenfalls erstrebenswert. Ein solcher Ansatz sorgt nicht nur für körperlich und geistig gesunde Mitarbeiter, sondern auch für die gewünschten Produktivitätseffekte.

Insgesamt ist der Trend gesamtgesellschaftlich, alte Strukturen brechen auf, die Angst und Unsicherheit wächst (Luhmann 1997). Besonders in den großen Unternehmen sind aufgrund ihrer globalen Ausrichtung betriebsbedingte Neuausrichtungen der Personalentwicklung an der Tagesordnung. Firmenteile werden verkauft, aufgelöst, integriert und assimiliert. Eine Antwort darauf ist Spezialisierung. Die Wissenschaft bekommt den Auftrag und spezialisiert sich entsprechend auf die Untersuchung der Effekte einer alternden Belegschaft. Es müssen also Erkenntnisse zur wirtschaftlichen und sozial verträglichen Integration der Individuen in die sich verändernden Arbeitsstrukturen geliefert werden. Genau darauf ist zum Beispiel das WISE Demografie-Netzwerk ausgelegt. Es generiert aus transdisziplinärer Sicht Lösungen und Werkzeuge, um den demografischen Wandel nachhaltig zu meistern.

\section{Literatur}

Bamberger, M. / Rugh, J. / Mabry, L.: Real World Evaluation. Working Under Budget, Time, Data and Political Constraints. Thousand Oaks 2006.

Cramer, J. / Heijden, A. v. d. / Jonker, J.: Corporate social responsibility: making sense through thinking and acting. In: Business Ethics: A European Review 15, 4/2006, S. 380-389.

Dover, P. A.: Change agents at work: Lessons from Siemens Nixdorf. In: Journal of Change Management 3, 3/2003, S. 243.

Kistler, E.: Vom Aktionismus zur nachhaltigen Umsetzung. In: Demographic Fitness News 2/2007, S. 1.

Linz, M.: Was wird dann aus der Wirtschaft? Über Suffizienz, Wirtschaftswachstum und Arbeitslosigkeit. Wuppertal 2006.

Luhmann, N.: Die Gesellschaft der Gesellschaft. Frankfurt a. M. 1997.

Otto, S.: Bedeutung und Verwendung der Begriffe nachhaltige Entwicklung und Nachhaltigkeit Eine empirische Studie. Bremen 2007.

Voelpel, S. / Leibold, M. / Früchtenicht, J.-D.: Herausforderung 50 plus. Konzepte zum Management der Aging Workforce: Die Antwort auf das demographische Dilemma. Erlangen / New York 2007.

\section{【 AUTOREN + KONTAKT}

Dr. Sven Voelpel ist Direktor der WISE Research Group und Professor am Jacobs Center for Lifelong Learning and Institutional Development an der Jacobs University Bremen.

Dr. Siegmar Otto promovierte 2007 an der Jacobs Universität zum Thema Bedeutung und Verwendung der Begriffe nachhaltige Entwicklung und Nachhaltigkeit.

Jacobs University, Campus Ring 1, 28759 Bremen. Tel.: +49 4212004791 , E-Mail: voelpel@jacobs-university.de, siegmar.otto@gmail.com

Internet: http://www.jacobs-university.de/ directory/03040, http://www.wiseresearch.org 
(c) 20I0 Authors; licensee IÖW and oekom verlag. This is an article distributed under the terms of the Creative Commons Attribution Non-Commercial No Derivates License (http://creativecommons.org/licenses/by-nc-nd/3.o/), which permits unrestricted use, distribution, and reproduction in any medium, provided the original work is properly cited. 